Systematic Review

\title{
Local Infiltration Analgesia Versus Regional Blockade for Postoperative Analgesia in Total Knee Arthroplasty: A Meta-analysis of Randomized Controlled Trials
}

\footnotetext{
Bin $\mathrm{Hu}, \mathrm{MD}^{1}$, Tiao Lin, MD, PhD², Shi-gui Yan, MD³, Song-lin Tong, MD¹, Jian-hao Yu, MD, Jian-jie $\mathrm{Xu}, \mathrm{MD}^{1}$, and Yi-ming Ying, MD ${ }^{1}$

From: ${ }^{2}$ Department of Orthopaedic Surgery, Affiliated Cixi Hospital, Wenzhou Medical University, No. 999 Nanerhuandong Road, Ningbo 315300, People's Republic of China; ${ }^{2}$ Department of Orthopaedic Surgery, First Affiliated Hospital of Sun Yat-sen University, No.58 Zhongshan 2nd Road, Guangzhou 510080, People's Republic of China; 3Department of Orthopaedic Surgery, Second Affiliated Hospital, School of Medicine, Zhejiang University, No.88 Jiefang Road, Hangzhou 310009, People's Republic of China

Address Correspondence: Bin $\mathrm{Hu}, \mathrm{MD}$ Affiliated Cixi Hospital Wenzhou Medical university China E-mail: jointsahzu@aliyun.com

Disclaimer: The work was supported by the Zhejiang Provincial Medical and Health Science and Technology Project (2015117887), Natural Science Foundation of Science and Technology Department of Ningbo (2015A610218) National Natural Science Foundation of China (81171687, 81371954), and Scientific Research Fund of Zhejiang Provincial Education Department (Y201018936).

Manuscript received: 07-16-2015 Revised manuscript received: 12-17-2015 Accepted for publication: 12-17-2015

Free full manuscript: www.painphysicianjournal.com

Background: Total knee arthroplasty (TKA) is one of the most commonly performed procedures while postoperative analgesia still remains challenging. The efficacy and safety of local infiltration analgesia (LIA) versus regional blockade (RB; epidural analgesia and/or peripheral nerve block) for pain management after TKA are controversial.

Objectives: The purpose of this meta-analysis was to determine whether LIA compared with RB would provide better postoperative pain control, consume less morphine, facilitate early functional recovery, entail a differential risk of side effects and complications, and allow a shorter length of stay.

Study Design: This meta-analysis pooled all data published in randomized controlled trials (RCTs) examining the efficacy and safety of LIA versus RB following TKA.

Setting: The work was performed at Affiliated Cixi Hospital, Wenzhou Medical University.

Methods: Literature in English was searched using EMBASE, Medline, Cochrane Library, CINAHL, Web of Science, and Scopus from inception to April 2015. RCTs that compared LIA and RB for postoperative analgesia following TKA were included. Methodological quality was assessed using the Cochrane Back Review Group checklist, and a sensitivity analysis was performed. Sixteen RCTs with a total of 1,206 patients were finally included in our study.

Results: The results of our meta-analysis indicate that patients managed by LIA showed significantly lower numeric rating scale (NRS) score at rest (WMD: $-0.40[-0.72,-0.07] ; P=0.02$ ) when compared with those managed by RB. Difference of morphine consumption was not significant (WMD: -1.39 [-7.21, 4.44]; $P=0.64$ ) between the 2 groups. In terms of early functional recovery, the LIA group showed more straight leg raise (RR: $2.90[2.15,3.93] ; P<0.00001)$ on the first postoperative day; better range of motion within one week (WMD: 4.33 [2.61, 6.05]; $P<0.00001)$, but not at 3 months (WMD: $1.98[-0.02,3.98] ; P=0.05)$; and comparable knee society score (WMD: $-8.79[-27.05,9.48] ; P=0.35)$. Length of hospital stay of the LIA group was marginally shorter (WMD: $-0.25[-0.49,-0.01] ; P=0.05)$ than that of the RB group. Risk of side effects and complications were comparable between groups.

Limitations: The lack of a standard criterion regarding the technique details of LIA and heterogeneity resulting from the various analgesic components, dosages, and different administration methods might have posed a bias on the results.

Conclusion: Our results have indicated that LIA provided better analgesia than RB at rest and preserved quadriceps function in the immediate postoperative period, which may be beneficial to early functional recovery. And its safety profile is reliable. With the biases in our meta-analysis, a rigorous and adequately powered RCT is needed to validate our results.

Key words: Local infiltration analgesia, regional block, peripheral nerve block, epidural analgesia, postoperative analgesia, total knee arthroplasty, meta-analysis, randomized controlled trial

Pain Physician 2016; 19:205-214
} 
T otal knee arthroplasty (TKA) is a commonly performed procedure today while postoperative analgesia remains a challenging issue. It is reported that more than half of the patients undergoing total hip or knee replacement would experience severe pain in the early postoperative period (1). Considerable postoperative pain interferes with patients' participation in physiotherapy, prolongs inpatient stay, lowers patient satisfaction, and leads to chronic pain and dysfunction. Thus, effective pain control in the immediate postoperative period is crucial for patients' convalescence after TKA (1).

Several options are available for postoperative pain management following TKA, but all of them have shortcomings. Traditionally, the use of neuraxially administered analgesics or peripheral nerve blockade to control postoperative pain following major knee surgery is a well-established and widely accepted protocol. Epidural analgesia (EA) is a kind of neuraxially administrated analgesic regimen with widespread acceptance for postoperative pain control after TKA (2). It can provide a wide range of pain relief and is superior to that obtained with intravenous opioids (3). However, narcotic-related adverse effects such as nausea, vomiting, hypotension, urinary retention, pruritus, dizziness, somnolence, respiratory depression, and a risk of spinal infection or hematoma (in anticoagulated patients) have hindered its superiority $(2,4)$. Another form of regional blockade $(R B)$ is peripheral nerve block (PNB), which is commonly performed to control postoperative pain following major orthopedic surgeries. Peripheral block provides postoperative analgesia comparable, or even superior, to that obtained with EA or systematic opioids while associated with an improved side-effect profile, and is less likely to cause severe neuraxial complications $(5,6)$. Theoretically, it mainly includes femoral nerve block (FNB), sciatic nerve block, obturator nerve block, and adductor canal block for knee surgery. Among these, FNB is most commonly performed. However, delayed ambulation and accidental in-hospital fall associated with motor block have gradually aroused surgeons' attention $(7,8)$. Sciatic nerve block and obturator nerve block are usually used as adjuncts to FNB. Complications such as nerve damage and local infection are rare but not completely avoidable.

Local infiltration analgesia (LIA), as a multimodal analgesic protocol, has been the most dramatic progress for postoperative pain control following TKA in recent years. Busch et al (9) reported that intraoperative multimodal periarticular injection significantly reduced the postoperative analgesic consumption with improved patient satisfaction and no apparent risks over the first 24 hours. When compared with RB, several level I trials have shown LIA to be an effective alternative in controlling postoperative pain with a good safety profile and a low prevalence of adverse effects (10-12). In contrast, some concluded that LIA has been of equivocal benefit with variable results (13). The analgesic potential, functional benefit, and safety profile of LIA versus those commonly performed regional block protocols, such as EA and/or PNB, remain controversial.

To our knowledge, half of the qualified studies we retrieved have been included in 2 mixed systematic reviews $(14,15)$ that compared LIA with various analgesic methods after total hip and knee arthroplasty before 2012. During the past 2 years, many newly emerged randomized controlled trials (RCTs) $(11,12,16-22)$ focusing on this topic have been reported. To provide a more powerful recommendation, we pooled high-level evidence from RCTs to perform a meta-analysis comparing LIA only with RB (EA and/or PNB) in terms of postoperative pain control, morphine consumption, early functional recovery, side effects, complications, and length of in-hospital stay.

\section{Methods}

\section{Search Strategy}

We searched the electronic literature database of Embase, Medline, Cochrane Library, CINAHL, Web of Science, and Scopus for RCTs that investigated the efficacy and safety of LIA versus RB for postoperative pain control following TKA. The literature search was done in December 2014 and results retrieved were last updated in April 2015. The search term was as follows: "(periarticular anesthesia infiltration OR local infiltration analgesia OR intraarticular analgesia OR regional analgesia) AND (epidural analgesia OR nerve block) AND (knee arthroplasty OR knee replacement)." Bibliographies of all the relevant retrieved articles were scrutinized to identify any additional studies of interest.

\section{Inclusion and Exclusion Criteria}

The meta-analysis was performed according to the Preferred Reporting Items for Systematic Reviews and Meta-analysis (PRISMA) guidelines (23). Studies were included if they met the following criteria: (i) RCTs investigating postoperative analgesia of LIA versus RB following primary TKA, (ii) English language, (iii) a minimum sample size of 10 patients at baseline, (iv) a minimum 
follow-up of 24 hours, (v) reported at least one of the following outcome measures: postoperative pain at rest or exercise, early functional recovery, morphine consumption, incidence of side effects, complication rate, length of in-hospital stay. Studies were excluded if they (i) were nonrandomized trials, (ii) contained concurrent LIA and RB in one group, (iii) were review articles, case reports, technique notes, editorials, letters, commentaries, (iv) comparative study without clinical relevant data or without explicitly stated inclusion criteria, (v) did not contain any of the above outcomes.

\section{Data Management}

The methodological quality of the included studies were assessed by 2 reviewers independently according to the method described by Furlan et al (24), which contained assessing factors such as randomization, allocation concealment, selective reporting, similar baseline, patient compliance, blinding, and co-intervention. Each item was scored as positive (+), negative (-), or unclear (?). The total score was computed by counting the number of items scored as positive. By this standard, the maximum quality score given to a study is 12 points and those with a score of $\geq 8$ points were considered to be of high quality, indicating low risk of bias.

For each eligible study, 2 reviewers independently extracted relevant data from the LIA group and the RB group. The outcome measurements were numeric rating scale (NRS) pain score at rest and/or motion, NRS pain score at different follow-ups within the first 24 hours, postoperative morphine consumption, early functional recovery including range of motion (ROM), straight leg raise (SLR), and knee society score, side effects and complications, and in-hospital stay. If useful data were reported only in graphic plots, we quantified them using plot-digitizing software (Plot Digitizer Version 2.6.4, Joseph Huwaldt and Scott Steinhorst). When collecting data of NRS score at different follow-ups, the data of rest were abstracted if both rest and motion NRS score were available. When nausea and vomiting were reported at several time points, we used the maximal value in each group. The data only for vomiting was used when both nausea and vomiting were reported (5). For in-hospital stay, we abstracted the real duration of stay, not the time to readiness for discharge. For studies that provided no extension data on knee movement, we estimated the ROM using flexion data. In terms of infection, urinary tract infection and other infection unrelated to the surgery itself were omitted. Two studies $(25,26)$ provided data of morphine consumption for the first postoperative day while we were not sure if those were the data of the first 24 hours.

\section{Statistical Analysis}

Pooled analysis was performed to compare the outcome measurements between groups using Review Manager (Revman Version 5.1.6., The Cochrane Collaboration, Oxford, UK) and STATA 12.0. Continuous data were expressed as weighted mean difference (WMD) with a $95 \%$ confidence interval $(\mathrm{Cl})$, and the dichotomous were expressed as relative risk (RR) with a $95 \%$ $\mathrm{Cl}$. We considered it as substantial heterogeneity when I $2>50 \%$, and in this situation, a random-effects model was used. A funnel plot was constructed to assess the potential influence of publication bias on the results. When heterogeneity was considered substantial, its origin was explored by performing pre-specified subgroup analyses where applicable.

\section{Results}

\section{Study Identification and Selection}

The literature search initially yielded 801 relevant articles from the 6 databases and bibliographies in all, of which 272 were excluded as duplicates. The titles and abstracts of remaining 529 articles were screened to determine their eligibility, and we excluded 490 that were irrelevant to the topic of interest. The full text of the 39 remaining studies was retrieved, and 23 of them did not fulfill the inclusion and exclusion criteria. Finally, 16 RCTs (10-12,16-22,25-30) were included in the present meta-analysis (Fig. 1). Among these, 5 $(17,20,21,27,29)$ were designed to compare LIA with $E A$, one (20) was designed to compare LIA with EA plus PNB, and $10(10,12,16,18,19,22,25,26,28,30)$ were designed to compare LIA with PNB. A total of 1,206 patients were enrolled. The mean age ranged from 66 to 76. All the studies were published from 2007 to 2015. The adopted form of PNB in these studies was exclusively FNB, and it was performed based on certain landmarks with nerve stimulator or ultrasound-guided technique. The specific protocols/details of LIA varied among studies, but the main sites of local infiltrations including the capsule, the retinacular tissue, and the subcutis were approximately the same. The morphine consumption of 2 studies $(25,26)$ was calculated by days rather than hours. They were excluded when performing subgroup analysis because the first 24 hour morphine consumption could not be figured out precisely. Details of study characteristics were summarized 
Relevant literatures in total $(n=801)$

Web of Science (286) Medline (185)

Scopus (147) Cochrane Library (113)

CINAHL (17) EMBASE (47)

Manual search of reference lists (6)

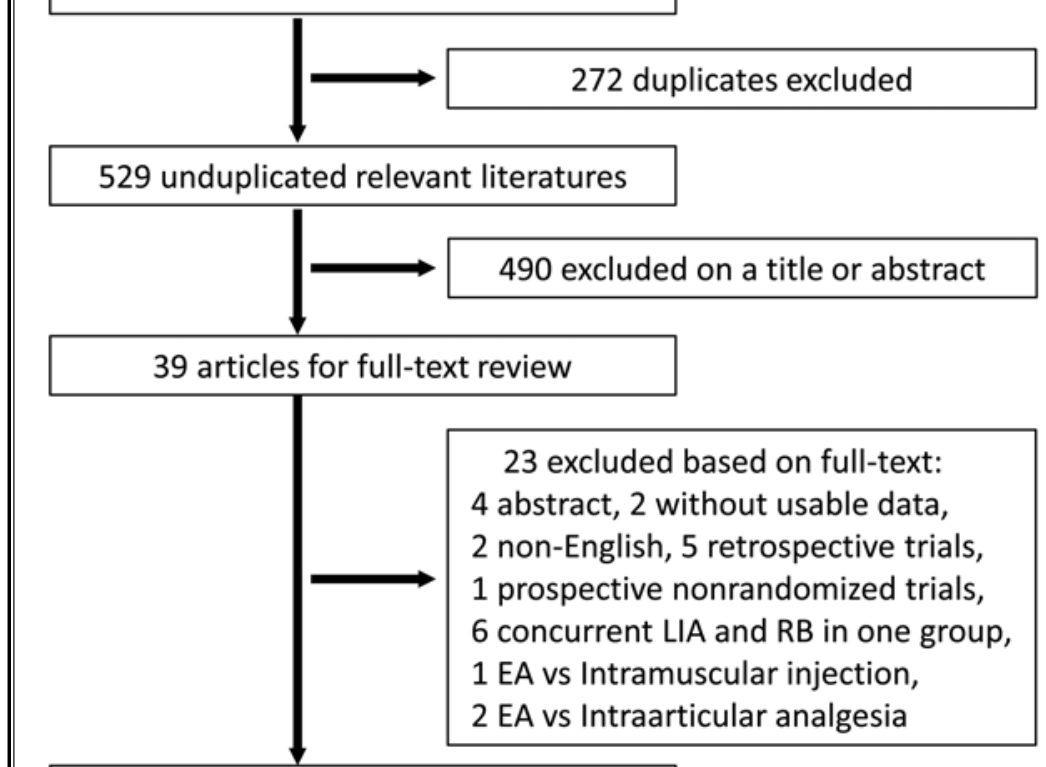

16 articles included in final analysis

Fig. 1. A flow diagram illustrates the retrieval of studies.

in Table 1. After assessment, methodological qualities of 11 studies (10$12,16,18,19,22,25-27,30)$ were categorized as high, which indicated low risk of bias. Qualities of the remaining 5 studies $(17,20,21,28,29)$ were categorized as moderate.

\section{Postoperative Pain}

When data of all studies were pooled, significant difference was detected in NRS pain score between LIA and RB at rest (WMD: -0.40 [-0.72, -0.07]; $P=0.02$ ) while no significant difference was detected regarding NRS score between the 2 groups at motion (WMD: -0.21 [-0.76, 0.33]; $P$ $=0.44$ ) (Fig. 2). After conducting a sensitivity analysis for NRS score at rest, the direction of effect was not changed by omitting any one of the studies. But for NRS score at motion, the direction of effect was changed when excluding the data of Andersen et al (27) in the sensitivity analysis and heterogeneity also decreased dramatically (WMD: 0.21 [-0.02, 0.45]; $P=0.07)$. Although it still didn't indicate significant difference in NRS score at motion between the 2 groups. Subgroup analysis revealed that NRS score at rest in the LIA group was significantly lower than that in the EA group (WMD: -0.89 [-1.43, -0.35$] ; P=0.001$ ), while comparable to that of PNB. When compared with LIA at motion, a tendency towards lower NRS scores was detected in the PNB group (WMD: 0.25 [-0.02, 0.53]; $P=$ 0.07 ). Difference of effects of LIA versus EA on NRS score at motion was not significant.

Postoperative NRS score at 4 hours (WMD: -1.25 [-2.51, 0.00]; $P$ $=0.05$ ) was marginal lower in the LIA group, and there is no statistical significance detected at 12 hours (WMD: $0.12[-0.36,0.61] ; P=0.62$ ) between the 2 groups. The NRS score of the LIA group at 8 hours (WMD: -1.01 [-1.91, -0.11]; $P=0.03$ ), 24 hours (WMD: -0.51 [-0.94, -0.09]; $P=0.02)$, and the overall effect of different follow-ups ( $P=0.001)$ was significantly lower than that of the RB group.

\section{Morphine Consumption}

Morphine consumption of first 24 hours was similar between the LIA group and RB group (WMD: -1.39 [-7.21, 4.44]; $P=0.64$ ) (Fig. 3). Due to the existence of substantial heterogeneity, we conducted subgroup analysis after excluding studies which did not calculate morphine consumption by hours. It revealed that significantly less morphine was consumed by patients of the LIA group than the PNB group (WMD: $-5.80[-9.58,-2.03] ; P=0.003)$.

\section{Early Functional Recovery}

Straight leg raise (SLR) on the first postoperative day was reported in 3 studies $(18,22,28)$, the results showed that more patients in the LIA group can perform SLR on first postoperative day (RR: 2.90 [2.15, 3.93]; $P<0.00001$ ) (Fig. 4). Shortterm (within one week) range of motion (ROM) of the LIA group was significantly higher than that of the RB group (WMD: 4.33 [2.61, 6.05]; 
Table1. Overview of characteristics of included studies.

\begin{tabular}{|c|c|c|c|c|c|c|}
\hline Study & $\begin{array}{l}\text { Patients } \\
\text { No. }\end{array}$ & Age & $\begin{array}{c}\text { Local infiltration } \\
\text { analgesia }\end{array}$ & Regional blockade & Anesthesia & Approach \\
\hline \multicolumn{7}{|c|}{ Local infiltration analgesia vs. Epidural analgesia } \\
\hline Andersen et al. 2010 & 49 & 67.9 & LIA & EA & Spinal & NA \\
\hline Binici Bedir et al. 2014 & 30 & 69.4 & LIA & EA & Spinal & Median parapatellar \\
\hline Spreng et al. 2010 & 102 & 66.5 & $\begin{array}{l}\text { LIA plus intraarticular } \\
\text { anesthetics }\end{array}$ & EA & Spinal & NA \\
\hline Tsukada et al 2014 & 111 & NA & LIA & EA & Spinal & $\begin{array}{c}\text { Subvastus approach/ } \\
\text { lateral }\end{array}$ \\
\hline Tsukada et al. 2015 & $71 \#$ & 72.7 & LIA & EA & Spinal & $\begin{array}{c}\text { Subvastus approach } \\
\text { lateral }\end{array}$ \\
\hline \multicolumn{7}{|c|}{ Local infiltration analgesia vs. Epidural analgesia + Peripheral nerve block } \\
\hline Yadeau et al. 2013 & 91 & 66 & LIA & EA plus single FNB & Spinal & Median parapatellar \\
\hline \multicolumn{7}{|c|}{ Local infiltration analgesia vs. Peripheral nerve block } \\
\hline Affas et al. 2011 & 40 & 68 & $\begin{array}{l}\text { LIA plus intraarticular } \\
\text { anesthetics }\end{array}$ & continuous FNB & Spinal & NA \\
\hline Ashraf et al. 2013 & 42 & NA & LIA & single FNB & Spinal & NA \\
\hline Carli et al. 2010 & 40 & 70.9 & $\begin{array}{l}\text { LIA plus intraarticular } \\
\text { anesthetics }\end{array}$ & $\begin{array}{c}\text { continuous FNB plus } \\
\text { posterior capsule } \\
\text { infiltration }\end{array}$ & Spinal & Median parapatellar \\
\hline Chaumeron et al. 2013 & 60 & 66.9 & $\begin{array}{l}\text { LIA plus intraarticular } \\
\text { anesthetics }\end{array}$ & continuous FNB & Spinal & Median parapatellar \\
\hline Moghtadaei et al. 2014 & 40 & 65.7 & LIA & single FNB & Spinal & Median parapatellar \\
\hline Ng et al. 2012 & $18 \#$ & 70 & LIA & continuous FNB & General & Median parapatellar \\
\hline Parvataneni et al. 2007 & 60 & 69.5 & LIA & single FNB & Spinal & Median parapatellar \\
\hline Spangehl et al. 2015 & 162 & 67.7 & LIA & $\begin{array}{c}\text { continuous FNB plus } \\
\text { single sciatic nerve } \\
\text { block }\end{array}$ & General & Medial parapatellar \\
\hline Toftdahl et al. 2007 & 80 & 70.9 & $\begin{array}{l}\text { LIA plus intraarticular } \\
\text { anesthetics }\end{array}$ & $\begin{array}{c}\text { continuous FNB } \\
\text { plus intraarticular } \\
\text { anesthetics }\end{array}$ & Spinal & Median parapatellar \\
\hline Uesugi et al. 2014 & 210 & 76.2 & LIA & $\begin{array}{l}\text { single FNB plus single } \\
\text { sciatic nerve block }\end{array}$ & Spinal & Midvastus \\
\hline
\end{tabular}

LIA, local infiltration analgesia; EA, epidural analgesia; FNB, femoral nerve block; NA, not applicable; \#, bilateral total knee arthroplasty.

$P<0.00001)$, and long-term (1.5 - 6 months) ROM of the LIA group was marginal higher than that of the RB group (WMD: 1.98 [-0.02, 3.98]; $P=0.05$ ). Knee society score, one measured at 6 weeks and one at 12 months, was also similar between the 2 groups (WMD: -8.79 $[-27.05,9.48] ; P=0.35)$. Four studies reported walking capacity of the early postoperative days but the measures varied. One (25) reported similar results by $2 \mathrm{~min}$ - ute walk test, one (18) reported a longer daily walking distance in patients of the LIA group on postoperative days 0,2 , and 3 , and $2(29,30)$ stated that the ability to walk > 3 meters was better in the LIA group.

\section{Side Effects and Complications}

Twelve studies provided data on postoperative nausea and vomiting (PONV), 7 provided data on deep 
Pain Physician: May/June 2016: 19:205-214

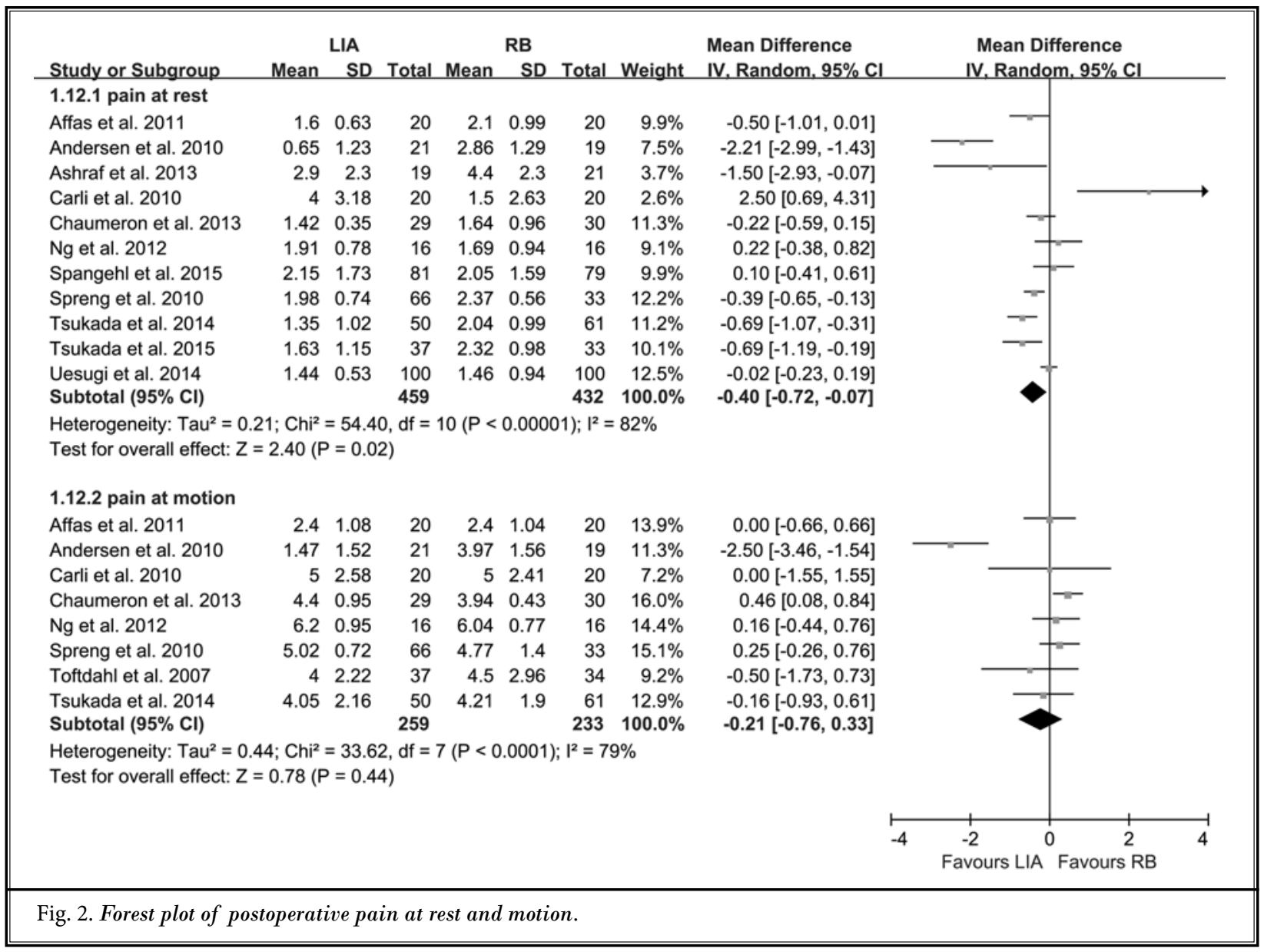

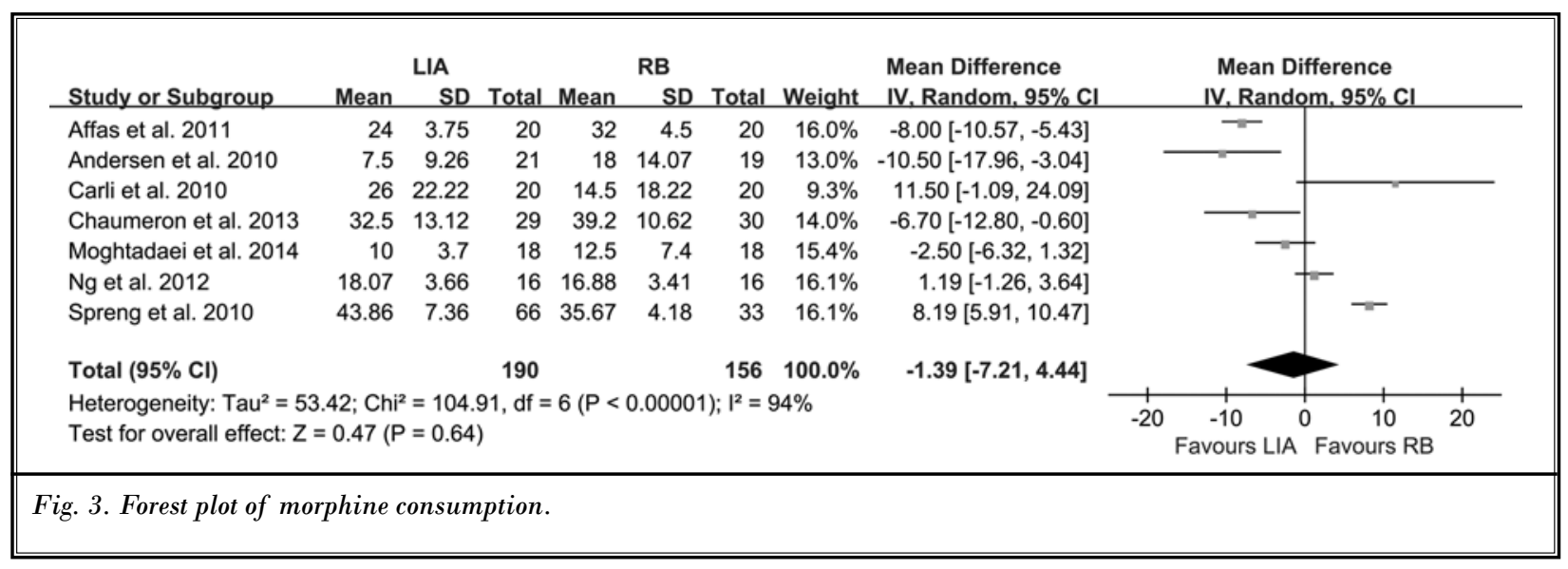

vein thrombosis (DVT), 11 provided data on wound complication, 3 provided data on in-hospital fall, and 11 provided data on infection. One pseudomonas infection occurred in the LIA group due to a sham femoral catheter and was not counted (18). The difference between the LIA group and $\mathrm{RB}$ group regarding the incidence of PONV (RR: 0.59 [0.34, 1.03]; $P=0.06$ ), DVT (RR: 0.34 $[0.09,1.34] ; P=0.12$ ), wound complication (RR: 1.68 $[0.69,4.05] ; P=0.25)$, in-hospital fall (RR: 0.20 [0.02, 1.67]; $P=0.14$ ), or infection (RR: $0.79[0.28,2.26] ; P=$ 


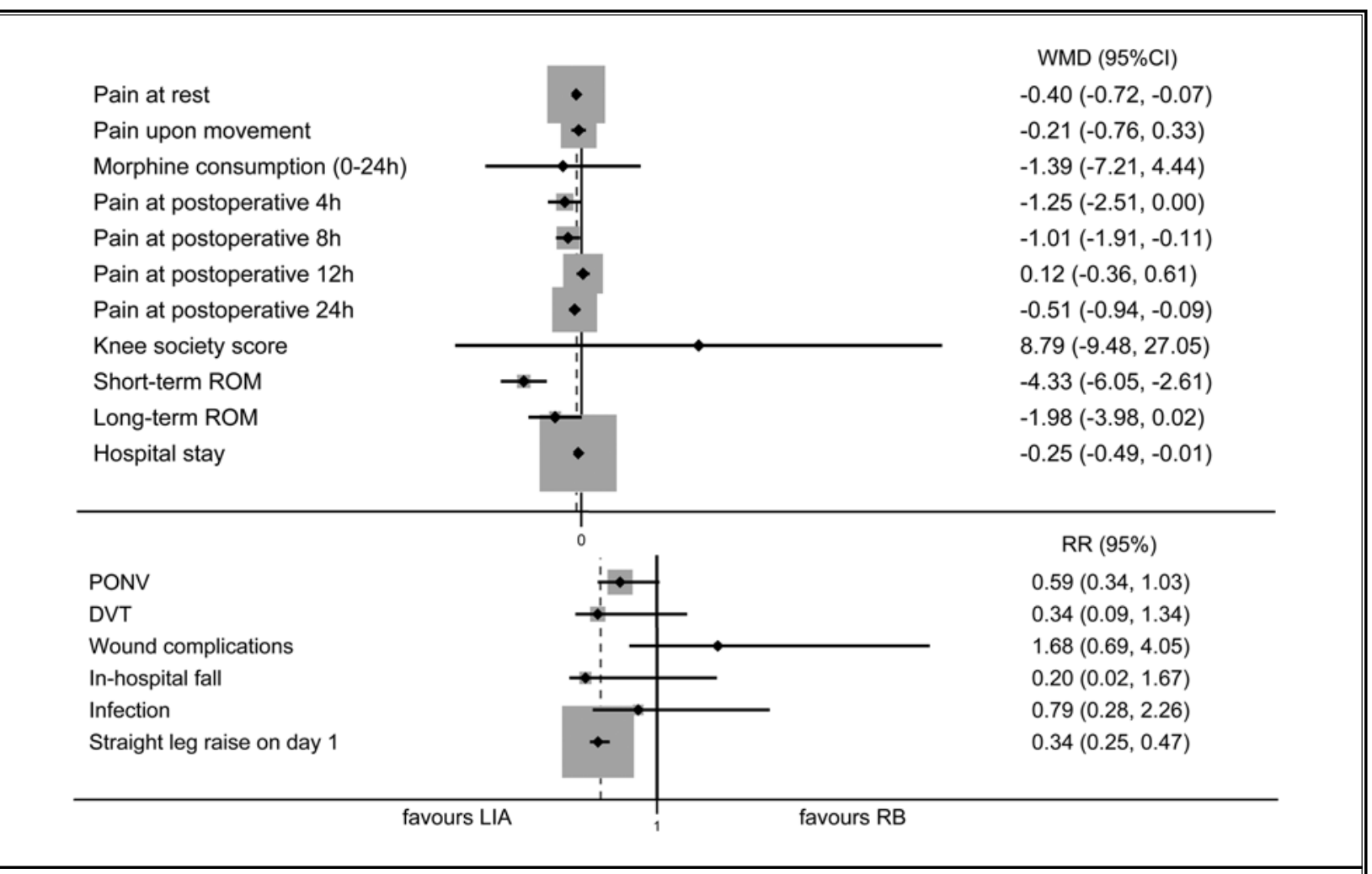

Fig. 4. Forest plot diagram showing a summary of the results of the meta-analysis.

0.66) were not statistically significant. A trend towards lower prevalence of PONV and DVT in the LIA group was observed. Subgroup analysis revealed a significantly lower incidence of PONV in the LIA group than the EA group (RR: 0.37 [0.15, 0.88]; $P=0.03$ ).

\section{In-hospital Stay}

For analysis of in-hospital stay, data from 2 studies $(19,29)$ which adapted the time to readiness for discharge for measuring length of stay were not included. The results indicated that LIA reduced the length of stay marginally (WMD: $-0.25[-0.49,-0.01] ; P=0.05$ ).

\section{Discussion}

Postoperative analgesia following TKA is critical to patients' rehabilitation and has become the focus of recent research $(11,12)$. Several effective protocols are available, but each has its own drawbacks. The analgesic potential, functional benefit, and safety profile of LIA versus RB protocols of proven analgesic effects, such as EA and/or PNB, remain controversial. Thus, we conducted a meta-analysis to determine whether LIA, compared with RB, provided better postoperative pain control, consumed less morphine, facilitated early functional recovery, allowed a shorter length of stay, and entailed a differential risk of side effects and complications following primary TKA.

The present study showed that LIA provides better postoperative analgesia than RB at rest. Results of subgroup analysis favors LIA over EA regarding the rest NRS score, but effects of LIA and PNB were comparable. As to NRS score at motion, no significant difference between LIA and RB was observed. The NRS values in the early postoperative period fluctuated and some factors may account for this. First, the result of the 4 hour NRS score could be partially influenced by the residual effects of surgical anesthesia. In other words, effects of regional block at this time point may be covered to some extent by the effects of intraoperative opioids. Second, different pharmacokinetics of the narcotics may slightly contribute to the results at different follow-ups. Another finding of our meta-analysis is that postoperative morphine consumption of LIA is comparable to that of RB, while the LIA group was 
found to consume significantly less morphine than the PNB group did. However, the population enrolled was relatively small and the results may not be conclusive.

In terms of functional recovery, the results suggested that more patients in the LIA group were able to perform SLR on the first postoperative day despite a relatively small population enrolled $(n=265)$. Similarly, short-term ROM (within one week) was found to be improved significantly with the LIA technique. These may not be an indicator of favorable pain control in the LIA group. It is more likely attributed to the absence of motor block. As a result, early functional recovery will be strengthened by functional quadriceps. Although the difference was marginal between the 2 groups, patients managed by LIA also showed a tendency towards improved long-term ROM (1.5 - 6 months) when compared with the RB group. In general, the LIA technique is beneficial to the early functional recovery following total knee replacement. The present results did indicate that LIA led to a marginal reduction in the length of hospital stay. Besides the analgesic technique, many factors affect the length of stay after knee replacement. Therefore we did not adapt the time to readiness for discharge for measuring length of stay. And also for this reason, the result regarding length of stay is not conclusive and of less clinical significance.

No statistically significant difference was detected between the LIA group and the RB group regarding the rates of side effects and complications. For PONV, the LIA technique showed a tendency towards decreased incidence with a moderate heterogeneity (66\%). Subgroup analysis revealed a statistically significant lower incidence of PONV in the LIA group than in the EA group, but PONV is comparable between LIA and PNB. This was consistent with the results of previous reviews $(14,15)$. Less DVT occurred in the LIA group than the RB group according to the pooled data, though the difference was not significant. Early participation in physiotherapy, which was facilitated in the LIA group, might help reduce venous stasis and prevent the incidence of vein thrombosis to a certain extent (18) despite that both groups were treated with some kind of anticoagulant. In terms of wound complication and infection, incidences were comparable between groups and kept at low level. Four events of in-hospital fall happened in the RB group while none happened in the LIA group. This uncommon event finally resulted in surgical intervention in one patient due to wound disruption during the fall (18) and lumbar vertebral fracture in one patient (22). Consequently, some have raised safety concerns about PNB for postoperative analgesia TKA $(8,31)$. Further investigations on modified protocols are required to handle the quadricep weakness and motor block following PNB.

Compared with RB, LIA is technically less difficult and requires less training and practice before expertise is acquired. Patients who receive RB might experience more motor block, thus making early ambulation suboptimal and increasing the risk of in-hospital fall, though this happens in rare cases. As epinephrine is useful for maintaining tissue levels of anesthetic and has the additional benefit of decreasing bleeding (32), it is sometimes supplemented as an adjunct to "cocktail" injections. However, care should be taken to avoid injecting epinephrine too close to the dermis since a case of wound necrosis has been documented (30).

Recently, there has been a shifting trend from the use of FNB towards the use of adductor canal block for postoperative analgesia in patients undergoing TKA. The saphenous nerve, the anterior branch of obturator nerve, and a branch of the posterior femoral nerve within the adductor canal are sensory to the surrounding tissue of the knee (33). As the FNB is associated with impaired quadriceps muscle strength, it seems to be an enticing strategy to block at the level of adductor canal without producing quadriceps weakness (34). Recent trials have achieved expected results $(35,36)$ and this may potentially benefit the outcome of physical therapy. However, we've noticed that the local anesthetic volumes adopted in these trials were much greater than an effective adductor canal block needed (37). It can lead to the spread of injectate both proximally (38) and distally (39), making the results of this newer technique unpredictable. On the other hand, the most important cause of quadriceps and hamstring muscle dysfunction after TKA is the surgery itself (40), though no association was found between type of PNB and in-hospital fall (41). Generally, many aspects like local anesthetic volume and optimal block level should be fully addressed under the multimodal analgesic protocol. There is much work to be done before a possibly full transfer from FNB to adductor canal block. We acknowledge, at the same time, that encouraging results with maintenance of more quadriceps strength have been achieved with adductor canal block in primary TKA and we also expect to see the results of this critical advancement in other knee related surgeries.

There are several potential limitations to the present study. First, there has not been a standard criterion regarding the technique details of LIA. Results differ as 
the techniques vary, making it difficult to draw a powerful conclusion. Second, most studies used accumulating morphine consumption for periods of time, but it could be more accurate to adopt morphine totals for a short period like Chaumeron et al (18) did. The former method can conceal the fact that the benefit of one particular technique may be only limited to the several hours following surgery. Third, the number of patients recruited by some studies $(17,26)$ was relatively small, which may lead to overestimation of the effect of the intervention. Fourth, heterogeneity existed across studies inevitably due to the various components and dosages of analgesic and the different methods of administration (periarticular infiltration or with intra-articular infusion for LIA, continuous infusion or single injection for PNB). Further trials investigating different regimens of LIA and RB are needed. Besides, other high-quality RCTs presumably exist in non-English literature which could provide information regarding comparison of analgesic effects and/or functional results between the 2 groups.

\section{Conclusion}

The findings of our meta-analysis suggest that LIA provides better postoperative analgesia than RB at rest, especially when compared with EA. And preservation of quadriceps function in the immediate postoperative period might be advantageous in facilitating early mobilization and functional recovery. This issue has not been definitely determined and should be further confirmed by high quality trials. On the basis of current evidence, LIA could be recommended as an effective, safe, and economical alternative to conventional regional block techniques. In addition, adequately powered prospective randomized trials are needed to explore innovative multimodal regimens that enable a painless TKA with minimal unfavorable side effects.

\section{References}

1. Maheshwari AV, Blum YC, Shekhar L, Ranawat AS, Ranawat CS. Multimodal pain management after total hip and knee arthroplasty at the Ranawat Orthopaedic Center. Clin Orthop Relat Res 2009; 467:1418-1423.

2. Mahoney OM, Noble PC, Davidson J, Tullos HS. The effect of continuous epidural analgesia on postoperative pain, rehabilitation, and duration of hospitalization in total knee arthroplasty. Clin Orthop Relat Res 1990; 260:30-37.

3. Block BM, Liu SS, Rowlingson AJ, Cowan AR, Cowan JA, Jr., Wu CL. Efficacy of postoperative epidural analgesia: $A$ meta-analysis. JAMA 2003; 290:2455-2463.

4. Choi PT, Bhandari M, Scott J, Douketis J. Epidural analgesia for pain relief following hip or knee replacement. Cochrane Database Syst Rev 2003; 3:CDoo3071.

5. Fowler SJ, Symons J, Sabato S, Myles PS. Epidural analgesia compared with peripheral nerve blockade after major knee surgery: A systematic review and meta-analysis of randomized trials. $\mathrm{Br}]$ Anaesth 2008; 100:154-164.

6. Richman JM, Liu SS, Courpas G, Wong R, Rowlingson AJ, McGready J, Cohen $\mathrm{SR}, \mathrm{Wu} \mathrm{CL}$. Does continuous peripheral nerve block provide superior pain control to opioids? A meta-analysis. Anesth
Analg 2006; 102:248-257.

7. Feibel RJ, Dervin GF, Kim PR, Beaule $P E$. Major complications associated with femoral nerve catheters for knee arthroplasty: A word of caution. J Arthroplasty 2009; 24:132-137.

8. Ilfeld BM, Duke KB, Donohue MC. The association between lower extremity continuous peripheral nerve blocks and patient falls after knee and hip arthroplasty. Anesth Analg 2010; 111:1552-1554.

9. Busch CA, Shore BJ, Bhandari R, Ganapathy S, MacDonald SJ, Bourne RB, Rorabeck $\mathrm{CH}$, McCalden RW. Efficacy of periarticular multimodal drug injection in total knee arthroplasty - A randomized trial. ] Bone Joint Surg Am 2006; 88:959-963.

10. Ashraf A, Raut VV, Canty SJ, McLauchlan G). Pain control after primary total knee replacement. A prospective randomised controlled trial of local infiltration versus single shot femoral nerve block. The Knee 2013; 20:324-327.

11. Tsukada S, Wakui M, Hoshino A. Postoperative epidural analgesia compared with intraoperative periarticular injection for pain control following total knee arthroplasty under spinal anesthesia: A randomized controlled trial. J Bone Joint Surg Am 2014; 96:1433-1438.
12. Uesugi K, Kitano N, Kikuchi T, Sekiguchi M, Konno SI. Comparison of peripheral nerve block with periarticular injection analgesia after total knee arthroplasty: A randomized, controlled study. The Knee 2014; 21:848-852.

13. DeWeese FT, Akbari Z, Carline E. Pain control after knee arthroplasty - Intraarticular versus epidural anesthesia. Clin Orthop Relat Res 2001; 392:226-231.

14. Andersen LO, Kehlet H. Analgesic efficacy of local infiltration analgesia in hip and knee arthroplasty: A systematic review. Br J Anaesth 2014; 113:360-374.

15. Marques EM, Jones HE, Elvers KT, Pyke M, Blom AW, Beswick AD. Local anaesthetic infiltration for peri-operative pain control in total hip and knee replacement: Systematic review and meta-analyses of short- and long-term effectiveness. BMC Musculoskelet Disord 2014; 15:220.

16. Affas F, Nygårds EB, Stiller CO, Wretenberg $\mathrm{P}$, Olofsson C. Pain control after total knee arthroplasty: A randomized trial comparing local infiltration anesthesia and continuous femoral block. Acta Orthop 2011; 82:441-447.

17. Binici Bedir E, Kurtulmus T, Basyigit S, Bakir U, Saglam N, Saka G. A com- 
parison of epidural analgesia and local infiltration analgesia methods in pain control following total knee arthroplasty. Acta Orthop Traumatol Turc 2014; 48:73-79.

18. Chaumeron A, Audy D, Drolet P, Lavigne $M$, Vendittoli PA. Periarticular injection in knee arthroplasty improves quadriceps function. Clin Orthop Relat Res 2013; 471:2284-2295.

19. Moghtadaei M, Farahini H, Faiz SHR, Mokarami F, Safari S. Pain management for total knee arthroplasty: Single-injection femoral nerve block versus local infiltration analgesia. Iran Red Crescent Med J 2014; 16:e13247.

20. Yadeau JT, Goytizolo EA, Padgett DE, Liu SS, Mayman DJ, Ranawat AS, Rade MC, Westrich GH. Analgesia after total knee replacement: local infiltration versus epidural combined with a femoral nerve blockade: A prospective, randomised pragmatic trial. Bone Joint J 2013; 95-B:629-635.

21. Tsukada S, Wakui M, Hoshino A. Pain control after simultaneous bilateral total knee arthroplasty: A randomized controlled trial comparing periarticular injection and epidural analgesia. J Bone Joint Surg Am 2015; 97:367-373.

22. Spangehl MJ, Clarke HD, Hentz JG, Misra L, Blocher JL, Seamans DP. Periarticular injections and femoral \& sciatic blocks provide similar pain relief after TKA: A randomized clinical trial. Clin Orthop Relat Res 2015; 473:45-53.

23. Moher D, Liberati A, Tetzlaff J, Altman DG. Preferred reporting items for systematic reviews and meta-analyses: The PRISMA statement. Ann Intern Med 2009; 151:264-269.

24. Furlan AD, Pennick V, Bombardier C, van Tulder M. 2009 updated method guidelines for systematic reviews in the Cochrane Back Review Group. Spine (Phila Pa 1976) 2009; 34:1929-1941.

25. Carli F, Clemente A, Asenjo JF, Kim DJ, Mistraletti G, Gomarasca M, Morabito A, Tanzer M. Analgesia and functional outcome after total knee arthroplasty: Periarticular infiltration vs continuous femoral nerve block. Br J Anaesth 2010; 105:185-195.

26. Ng F-Y, Ng JK-F, Chiu K-Y, Yan C-H, Chan C-W. Multimodal periarticular injection vs continuous femoral nerve block after total knee arthroplasty: A prospective, crossover, randomized clinical trial. J Arthroplasty 2012; 27:1234-1238.

27. Andersen KV, Bak M, Christensen BV Harazuk J, Pedersen NA, Søballe K. A randomized, controlled trial comparing local infiltration analgesia with epidural infusion for total knee arthroplasty. Acta Orthop 2010; 81:606-610.

28. Parvataneni HK, Shah VP, Howard H, Cole N, Ranawat AS, Ranawat CS. Controlling pain after total hip and knee arthroplasty using a multimodal protocol with local periarticular injections: A prospective randomized study. J Arthroplasty 2007; 22:33-38.

29. Spreng UJ, Dahl V, Hjall A, Fagerland MW, Raeder J. High-volume local infiltration analgesia combined with intravenous or local ketorolac plus morphine compared with epidural analgesia after total knee arthroplasty. $\mathrm{Br}$ J Anaesth 2010; 105:675-682.

30. Toftdahl K, Nikolajsen L, Haraldsted V, Madsen F, Tønnesen EK, Søballe K. Comparison of peri- and intraarticular analgesia with femoral nerve block after total knee arthroplasty: A randomized clinical trial. Acta Orthop 2007; 78:172-179.

31. Sharma S, lorio R, Specht LM, DaviesLepie S, Healy WL. Complications of femoral nerve block for total knee arthroplasty. Clin Orthop Relat Res 2010; 468:135-140.

32. Anderson LA, Engel GM, Bruckner JD, Stoddard GJ, Peters CL. Reduced blood loss after total knee arthroplasty with local injection of bupivacaine and epinephrine. J Knee Surg 2009; 22:130-136.

33. Lund J, Jenstrup MT, Jaeger P, Sørensen AM, Dahl JB. Continuous adductor-ca- nal-blockade for adjuvant post-operative analgesia after major knee surgery: Preliminary results. Acta Anaesthesiol Scand 2011; 55:14-19.

34. Kim DH, Lin Y, Goytizolo EA, Kahn RL, Maalouf DB, Manohar A, Patt ML, Goon AK, Lee YY, Ma Y, Yadeau JT. Adductor canal block versus femoral nerve block for total knee arthroplasty: A prospective, randomized, controlled trial. Anesthesiology 2014; 120:540-550.

35. Ludwigson JL, Tillmans SD, Galgon RE, Chambers TA, Heiner JP, Schroeder KM. A comparison of single shot adductor canal block versus femoral nerve catheter for total knee arthroplasty. J Arthroplasty 2015; 30:68-71.

36. Grevstad U, Mathiesen O, Valentiner LS, Jaeger P, Hilsted KL, Dahl JB. Effect of adductor canal block versus femoral nerve block on quadriceps strength, mobilization, and pain after total knee arthroplasty: A randomized, blinded study. Reg Anesth Pain Med 2015; 40:3-10.

37. Egeler C, Jayakumar A, Ford S. Motor-sparing knee block - description of a new technique. Anaesthesia 2013; 68:542-543.

38. Chen J, Lesser JB, Hadzic A, Reiss W, Resta-Flarer F. Adductor canal block can result in motor block of the quadriceps muscle. Reg Anesth Pain Med 2014; 39:170-171.

39. Gautier PE, Lecoq JP, Vandepitte C, Harstein G, Brichant JF. Impairment of sciatic nerve function during adductor canal block. Reg Anesth Pain Med 2015; 40:85-89.

40. Stevens-Lapsley JE, Balter JE, Kohrt WM, Eckhoff DG. Quadriceps and hamstrings muscle dysfunction after total knee arthroplasty. Clin Orthop Relat Res 2010; 468:2460-2468.

41. Memtsoudis SG, Danninger T, Rasul R, Poeran J, Gerner P, Stundner O, Mariano ER, Mazumdar M. Inpatient falls after total knee arthroplasty: The role of anesthesia type and peripheral nerve blocks. Anesthesiology 2014; 120:551-563. 